\title{
Stress exposure and postural control in young females
}

\author{
MARINELLA COCO ${ }^{1}$, ANGELO SARRA FIORE ${ }^{1}$, VINCENZO PERCIAVALLE $^{1}$, TIZANA MACI $^{2}$, \\ MARIA CRISTINA PETRALIA ${ }^{1}$ and VALENTINA PERCIAVALLE ${ }^{3}$ \\ Departments of ${ }^{1}$ Biomedical Sciences - Physiology Section, ${ }^{2}$ 'G.F. Ingrassia' and ${ }^{3}$ Education Sciences, \\ University of Catania, Catania, Catania 95125, Italy
}

Received January 7, 2014; Accepted June 24, 2014

DOI: $10.3892 / \mathrm{mmr} .2014 .2898$

\begin{abstract}
The aim of this study was to determine if heightened stress had an adverse affect on the postural control of 14 young right-handed females during quiet standing in either the presence or the absence of visual input. The level of stress was evaluated by measuring the free cortisol response to awakening (cortisol awakening response; CAR) and by employing the perceived stress scale (PSS). Overall mood disturbance was measured using the profile of mood states (POMS). Postural control was evaluated using a force platform by measuring the $95 \%$ confidence ellipse area described by the center of pressure during 5 balance positions maintained for at least $52 \mathrm{sec}$, each with open and closed eyes. The results of this study revealed a significant positive correlation between CAR, PSS and POMS for each of the studied subjects. Furthermore, it was observed that whilst the level of stress was capable of influencing postural stability, this influence was particularly evident when no visual information was available. Additionally, it was determined that maintenance of posture is easier when the dominant foot is ahead, regardless of visual input.
\end{abstract}

\section{Introduction}

The ability to maintain a standing position is fundamental in gait and the initiation of rapid voluntary movements, the two of which are essential for undertaking everyday activities. This capability is commonly investigated through the traces of the center of pressure (COP). Several factors influence postural control, including the time of day (1-3), general and local fatigue (4), and age (5-7). It has been proposed that diminished

Correspondence to: Professor Vincenzo Perciavalle, Department of Biomedical Sciences - Physiology Section, University of Catania, 6 Viale Andrea Doria, Catania, Catania 95125, Italy

E-mail: perciava@unict.it

Abbreviations: COP, center of pressure; POMS, profile of mood states; PSS, perceived stress scale; A95, 95\% confidence ellipse area of COP; SD, standard deviation; TMD, total mood disturbance

Key words: stress, postural control, salivary cortisol, females central cognitive processing is primarily responsible for the impairment in balance performance with advancing age (8).

Emotions and mood can have a direct impact on the physical and mental health of an individual, such as sadness, apprehension, addiction and stress (9), and the physiological responses to stress are important determinants of overall health and susceptibility to disease (10). The consequences of stress can be either harmful or beneficial, depending on the intensity, duration, and frequency of the stress (11). It has been observed that high levels of stress are associated with worsening of executive functions, abstract reasoning, processing speed, and visual-spatial memory (10,12-14). Furthermore, it has been observed that mood states are capable of influencing balance control (15) and anticipatory postural adjustments (16).

The purpose of the present study was to assess whether heightened stress and negative mood experiences adversely affect the postural control in young females. The task undertaken by participants consisted of quiet standing in 5 positions selected from those proposed by Kirby et al (17): 3 of these positions had the feet on the same anteroposterior level but separated by different distances, whilst in the other 2 positions one of the feet was placed $10 \mathrm{~cm}$ in front of the other (in position $\mathrm{D}$, the dominant foot and in position $\mathrm{E}$, the non-dominant foot).

The perceived stress of the participants was assessed using the perceived stress scale (PSS), devised by Cohen et al (18), whilst overall mood disturbance was measured with the profile of mood states (POMS). As a second measure of stress, the cortisol awakening response (CAR) of participants was studied. The CAR is a rapid increase of free cortisol levels which occurs within 30 min of awakening and subsequently returns to baseline levels $\sim 1 \mathrm{~h}$ later (19). It has previously been reported that chronic stress has an important role in the CAR (as reviewed in 20,21); and so in the present study saliva samples were collected from participants in order to measure their CAR.

\section{Materials and methods}

Participants. A total of 14 healthy young females, students at the University of Catania (Catania, Italy), participated in this study. Only female participants were recruited for this study due to the gender-related differences in the brain structures that control the activity of the hypothalamic-pituitary-adrenal (HPA) axis, as well as differences in the levels of corticosteroid-binding 
globulins, which influence the basal and stress-induced activation of the HPA axis (as reviewed in 22). Participants were selected on the basis of similar anthropometric characteristics. The participants had a mean age of 23.79 years $( \pm 3.49)$, a mean height of $163.86 \mathrm{~cm}( \pm 4.88)$, a mean weight of $57.36 \mathrm{~kg}( \pm 3.77)$, a mean body mass index of $21.35( \pm 0.89)$ and all were right-handed. The dominant hand was determined by the Edinburgh Handedness Inventory (23). All the subjects signed informed consent documentation in accordance with the Ethical Committee of the University of Catania prior to their participation in the study.

Stress measurement. Participants' perceptions of stress were evaluated using the 10-item version of the PSS (18), which consists of 10 questions graded on a five-point Likert scale. Scores range from 0-40, with a higher score indicating greater subjective distress. The PSS is designed to allow individuals to rate how frequently they felt overwhelmed by stressful events during the previous 30 days, using a scale which ranges from 0 (never) to 4 (very often). One such question from the test is as follows: 'In the past week, how often have you felt difficulties were piling up so high that you could not overcome them?'. The mean score for normative females in the general population is $13.7 \pm 26.6(24)$.

Overall mood disturbance was evaluated using an abbreviated 30-item version of the POMS test developed by McNair et al (25). Participants rated each item on a 5 -point Likert scale with anchors ranging from 'Not at all' to 'Extremely'. Items were combined to form 6 separate subscales: Tension-anxiety (T), depression-dejection (D), anger-hostility (A), vigor-activity (V), fatigue-inertia (F), and confusion-bewilderment (C). For each of the 6 subscales, the raw scores were subjected to a $\mathrm{T}$-score transformation using the following formula: $\mathrm{T}=50+10(\mathrm{n}-\mathrm{m}) / \mathrm{s}$ where $\mathrm{n}=$ raw score, $\mathrm{m}=$ mean and $\mathrm{s}=$ standard deviation. This transformation converted the raw scores to scores on a standard scale with a mean value of $50 \pm 10$ (26). The 6 subscale T-scores were then be combined to form an overall measure of affect that is known as total mood disturbance $(\mathrm{TMD}=\mathrm{T}+\mathrm{D}+\mathrm{A}-\mathrm{V}+\mathrm{F}+\mathrm{C})$.

Salivary cortisol assay. Saliva samples were collected on two consecutive workdays, immediately upon awakening (sample 1), and $15 \mathrm{~min}$ (sample 2), $30 \mathrm{~min}$ (sample 3), 45 min (sample 4) and 60 min (sample 5) thereafter, resulting in five samples per day and a total of ten samples for each individual. Participants were requested to wake at the same time between 06.00 and 08:00 $\mathrm{h}$ on the two days and asked to remain in bed until all 5 saliva samples were obtained. Each sample was obtained by instructing participants to chew on a dental roll (Richmond Dental, Charlotte, NC, USA) for $\sim 1 \mathrm{~min}$ and then samples were stored in sterile containers which were provided. Samples were frozen at $-70^{\circ} \mathrm{C}$ until assay. All saliva profiles were sampled during the winter between January and February 2013. A radioimmunoassay cortisol test (AKS18EW; Radim SpA, Rome, Italy) was used to measure salivary free cortisol levels according to previously described methods (27).

Balance performance. COP excursion data were collected using an AMTI force platform (model OR-6-7-1000; AMTI, Newton, MA, USA). As shown in Fig. 1, COP measurements
Table I. Scores obtained in the 14 subjects.

\begin{tabular}{lccc}
\hline Subject & PSS & POMS - TMD & $\begin{array}{c}\text { Mean AUCr } \\
\text { (nmol/l) }\end{array}$ \\
\hline 1 & 12 & 143 & 20.63 \\
2 & 14 & 157 & 24.93 \\
3 & 24 & 239 & 22.46 \\
4 & 21 & 195 & 24.28 \\
5 & 16 & 169 & 26.74 \\
6 & 18 & 175 & 23.15 \\
7 & 19 & 184 & 24.27 \\
8 & 27 & 297 & 23.13 \\
9 & 22 & 198 & 23.24 \\
10 & 17 & 176 & 22.57 \\
11 & 14 & 168 & 23.37 \\
12 & 13 & 166 & 24.72 \\
13 & 23 & 164 & 22.23 \\
14 & 15 & 200 & 23.57 \\
Mean & 18.21 & 187.93 & 23.52 \\
SD & 4.59 & 39.14 & 1.45 \\
\hline
\end{tabular}

Mean values of salivary cortisol are expressed as the AUCr. PSS, perceived stress scale; POMS - TMD, profile of mood states expressed as total mood disturbance; AUCr, area under the response curve.

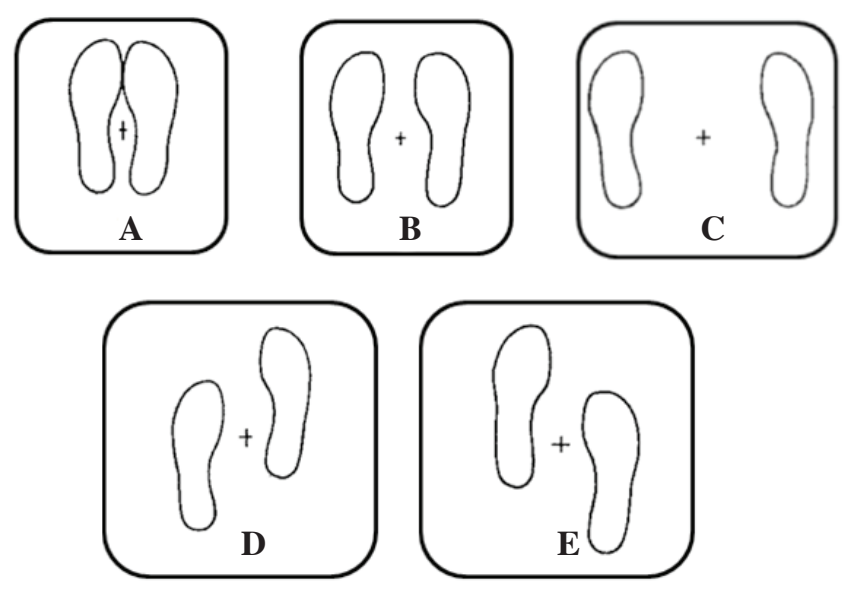

Figure 1. Balance positions used in the present study. (A) Feet together, (B) feet $15 \mathrm{~cm}$ apart, (C) feet $30 \mathrm{~cm}$ apart, (D) right foot forward $10 \mathrm{~cm}$, (E) left foot forward $10 \mathrm{~cm}$. Image adapted from Kirby et al (17).

were conducted in 5 balance positions adapted from a study by Kirby et al (17): (A) Feet together; (B) feet $15 \mathrm{~cm}$ apart; (C) feet $30 \mathrm{~cm}$ apart; (D) right foot forward $10 \mathrm{~cm}$; and (E) left foot forward $10 \mathrm{~cm}$. Each of these 5 positions was held for $52 \mathrm{sec}$ with eyes open and then $52 \mathrm{sec}$ with eyes closed.

To avoid influences of time of day on postural stability (1), the experiments were performed between 10:00 and 13:00 h. The force plate of the platform had a metal surface and all tests were conducted with subjects barefoot. The AMTI force platform simultaneously measures three force components along the $\mathrm{x}$ (medio-lateral); $\mathrm{y}$ (antero-posterior); $\mathrm{z}$ (vertical) axes and three moment components about the $\mathrm{x}-, \mathrm{y}$ - and $\mathrm{z}$-axes. Signals 
A

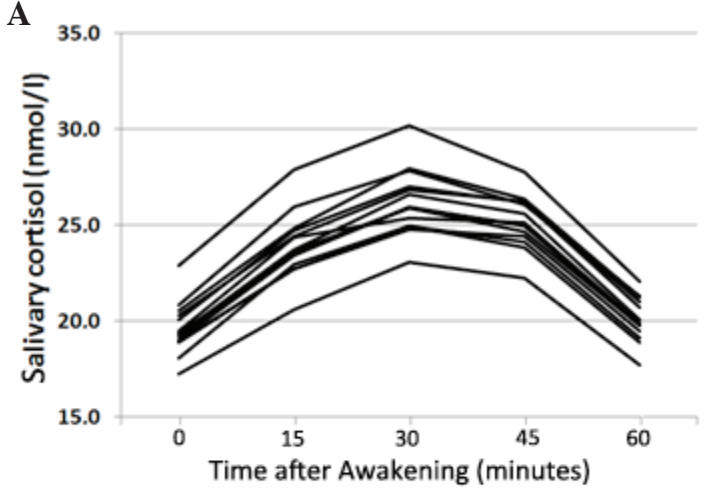

B

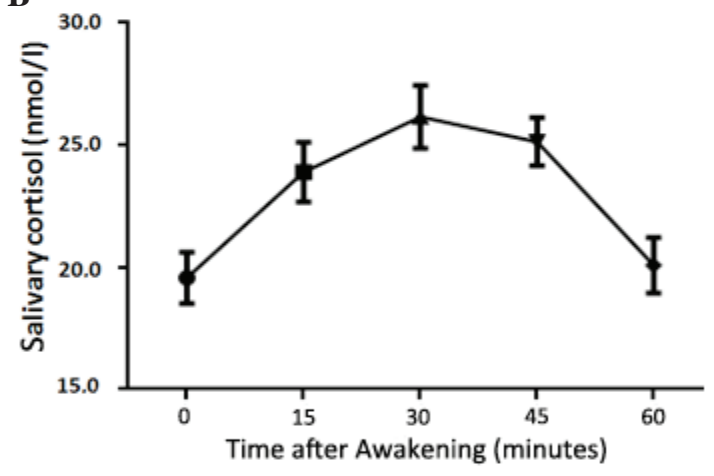

C

\begin{tabular}{|c|c|c|c|c|}
\hline & $\mathbf{1 5} \mathrm{min}$ & $\mathbf{3 0} \mathrm{min}$ & $\mathbf{4 5} \mathrm{min}$ & $\mathbf{6 0} \mathrm{min}$ \\
\hline $\mathbf{0 ~ m i n}$ & $* * *$ & $* * *$ & $* * *$ & $\mathrm{~ns}$ \\
\hline $\mathbf{1 5} \mathrm{min}$ & & $* * *$ & $*$ & $* * *$ \\
\hline $\mathbf{3 0} \mathrm{min}$ & & & $\mathrm{ns}$ & $* * *$ \\
\hline $\mathbf{4 5} \mathbf{~ m i n}$ & & & & $* * *$ \\
\hline
\end{tabular}

Figure 2. (A) Cortisol awakening profiles of each of the 14 subjects. (B) Mean values of cortisol awakening profiles. (C) Results of Tukey's multiple comparisons test between the mean values. ${ }^{*} \mathrm{P}<0.05,{ }^{* * * *} \mathrm{P}<0.001$. ns, not significant.

from the force platform were amplified through an AMTI MiniAmp MSA-6 Strain Gauge Amplifier system (AMTI) prior to being digitized into an IBM-compatible Pentium computer (Asus S550C; ASUSTeK Computer Inc., Taipei, Taiwan). The signals were digitized using a Cambridge Electronic Design 1401 acquisition unit (CED, Cambridge, England) at a sampling rate of $100 \mathrm{~Hz}$. Routines were developed with MATLAB software (The MathWorks Inc., Natick, MA, USA) to calculate the area corresponding to $95 \%$ of the area described by the COP trajectory (A95), since previous studies have demonstrated that this is a more sensitive measure of postural stability $(28,29)$.

Data analysis. All data are reported as the mean \pm standard deviation. For each of the 5 time points, cortisol levels were averaged over the 2 days to obtain a single mean cortisol level.

The cortisol increase was defined as the difference between the individual cortisol peak value (e.g., sample 2, 3, 4 or 5) and the cortisol level immediately after awakening (sample 1). The area under the response curve
A

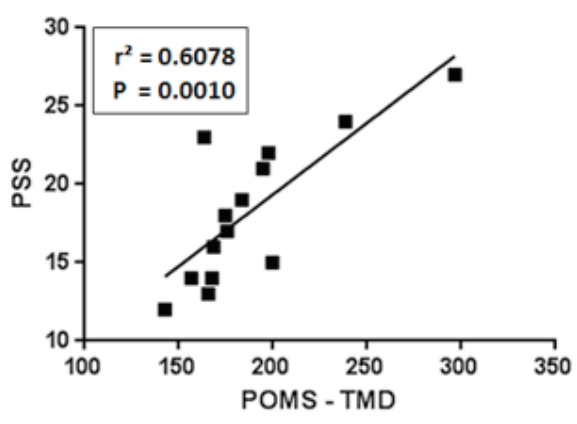

B

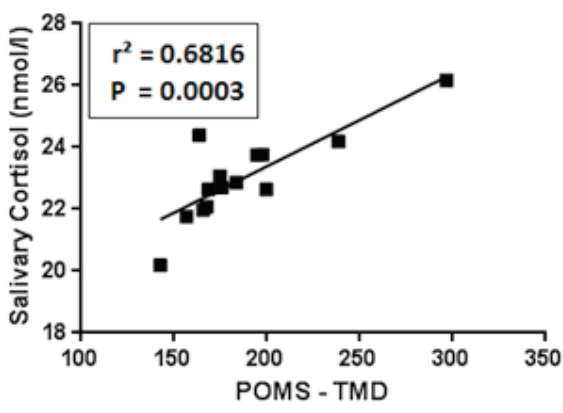

C

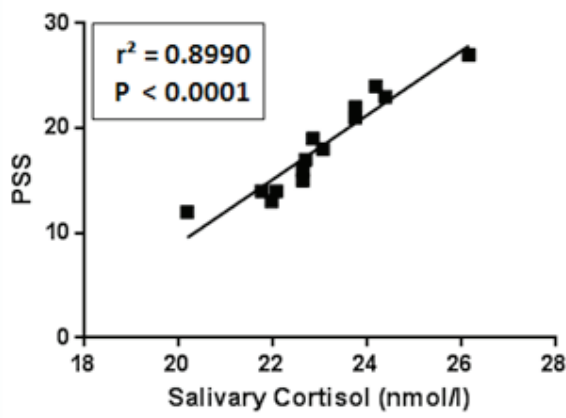

Figure 3. Correlations between profile of mood states expressed as total mood disturbance (POMS - TMD), perceived stress scale (PSS) and salivary cortisol for all of the studied subjects. (A) TMD and PSS, (B) TMD and salivary cortisol and (C) salivary cortisol and PSS. There is a significant positive correlation between all three variables.

(AUCr) was computed including individual baseline cortisol levels, as described in a previous study (30).

Data collected from the participants were compared by means of a non-parametric Wilcoxon signed-rank test. The correlation between variables was analyzed using a linear regression. Statistical analysis was performed according to the guidelines for reporting statistics in journals published by the American Physiological Society (31).

\section{Results}

PSS and TMD vary greatly between participants. Table I summarizes the results obtained for the mood measurements of all 14 subjects in the group. The level of stress experienced by participants varied greatly, the PSS ranged from 12 to 27 (mean: 18.21 \pm 4.59 ), TMD ranged from 143 to 297 (mean: $187.93 \pm 39.14$ ) and the salivary cortisol, expressed as area under the response curve (AUCr), ranged from 20.63 to $26.74 \mathrm{nmol} / 1$ (mean: $23.52 \mathrm{nmol} / 1 \pm 1.45$ ).

Salivary cortisol increases rapidly within 30 min of awakening. Fig. 2 shows the cortisol awakening profiles of the 
Table II. Area ( $95 \%$ confidence ellipse expressed in $\mathrm{cm}^{2}$ ) of the center of pressure measured in the 14 subjects when stood in each of the five balance positions (A-E) presented in Fig. 1.

\begin{tabular}{|c|c|c|c|c|c|c|c|c|c|c|c|c|}
\hline \multirow[b]{2}{*}{ Subjects } & \multicolumn{2}{|c|}{ A } & \multicolumn{2}{|c|}{ B } & \multicolumn{2}{|c|}{$\mathrm{C}$} & \multicolumn{2}{|c|}{$\mathrm{D}$} & \multicolumn{2}{|c|}{ E } & \multicolumn{2}{|c|}{ Mean value } \\
\hline & $\mathrm{OE}$ & $\mathrm{CE}$ & $\mathrm{OE}$ & $\mathrm{CE}$ & $\mathrm{OE}$ & $\mathrm{CE}$ & $\mathrm{OE}$ & $\mathrm{CE}$ & $\mathrm{OE}$ & $\mathrm{CE}$ & $\mathrm{OE}$ & $\mathrm{CE}$ \\
\hline 1 & 1.29 & 2.27 & 0.41 & 0.29 & 0.45 & 0.80 & 0.67 & 0.97 & 1.37 & 1.71 & 0.84 & 1.21 \\
\hline 2 & 1.13 & 1.38 & 1.59 & 3.58 & 2.88 & 0.80 & 2.24 & 2.38 & 3.84 & 4.05 & 2.34 & 2.44 \\
\hline 3 & 5.69 & 8.68 & 3.28 & 3.23 & 2.06 & 3.83 & 4.31 & 6.86 & 3.67 & 5.77 & 3.80 & 5.67 \\
\hline 4 & 5.06 & 4.60 & 3.84 & 1.71 & 1.52 & 1.69 & 1.95 & 2.01 & 3.53 & 3.57 & 3.18 & 2.72 \\
\hline 5 & 2.82 & 1.99 & 2.35 & 2.69 & 1.86 & 2.36 & 1.60 & 3.49 & 2.30 & 3.23 & 2.19 & 2.75 \\
\hline 6 & 3.11 & 4.96 & 1.39 & 2.37 & 1.05 & 0.77 & 3.46 & 3.61 & 1.41 & 1.80 & 2.08 & 2.70 \\
\hline 7 & 3.35 & 6.41 & 2.01 & 2.12 & 0.98 & 1.35 & 3.81 & 5.50 & 3.17 & 4.17 & 2.66 & 3.91 \\
\hline 8 & 0.10 & 5.87 & 1.35 & 1.53 & 2.28 & 2.36 & 6.37 & 13.35 & 4.61 & 6.98 & 2.94 & 6.02 \\
\hline 9 & 2.27 & 1.97 & 2.27 & 1.36 & 0.91 & 1.12 & 2.35 & 6.06 & 2.23 & 4.94 & 2.01 & 3.09 \\
\hline 10 & 2.93 & 2.85 & 2.18 & 2.27 & 1.20 & 0.98 & 2.88 & 3.51 & 1.66 & 1.95 & 2.17 & 2.31 \\
\hline 11 & 2.15 & 2.20 & 3.05 & 2.92 & 1.75 & 1.85 & 2.05 & 2.18 & 3.75 & 3.80 & 2.55 & 2.59 \\
\hline 12 & 1.44 & 1.97 & 1.05 & 1.15 & 0.80 & 0.80 & 1.02 & 1.15 & 1.58 & 1.82 & 1.18 & 1.38 \\
\hline 13 & 3.55 & 3.45 & 3.56 & 3.80 & 2.55 & 3.75 & 4.05 & 5.23 & 3.88 & 4.85 & 3.52 & 4.22 \\
\hline 14 & 1.55 & 1.60 & 1.78 & 2.22 & 2.45 & 2.35 & 2.54 & 2.66 & 3.54 & 4.56 & 2.37 & 2.68 \\
\hline Mean & 2.60 & 3.59 & 2.15 & 2.23 & 1.62 & 1.77 & 2.81 & 4.21 & 2.90 & 3.80 & 2.42 & 3.12 \\
\hline SD & 1.53 & 2.20 & 1.00 & 0.98 & 0.75 & 1.05 & 1.50 & 3.19 & 1.10 & 1.60 & 0.81 & 1.40 \\
\hline W-test & \multicolumn{2}{|c|}{ NS } & \multicolumn{2}{|c|}{ NS } & \multicolumn{2}{|c|}{ NS } & \multicolumn{2}{|c|}{$\mathrm{P}<0.05$} & \multicolumn{2}{|c|}{$\mathrm{P}<0.01$} & \multicolumn{2}{|c|}{$\mathrm{P}<0.05$} \\
\hline
\end{tabular}

OE, open eyes; CE, closed eyes; SD, standard deviation; W-test, Wilcoxon signed-rank test; NS, not significant.

A
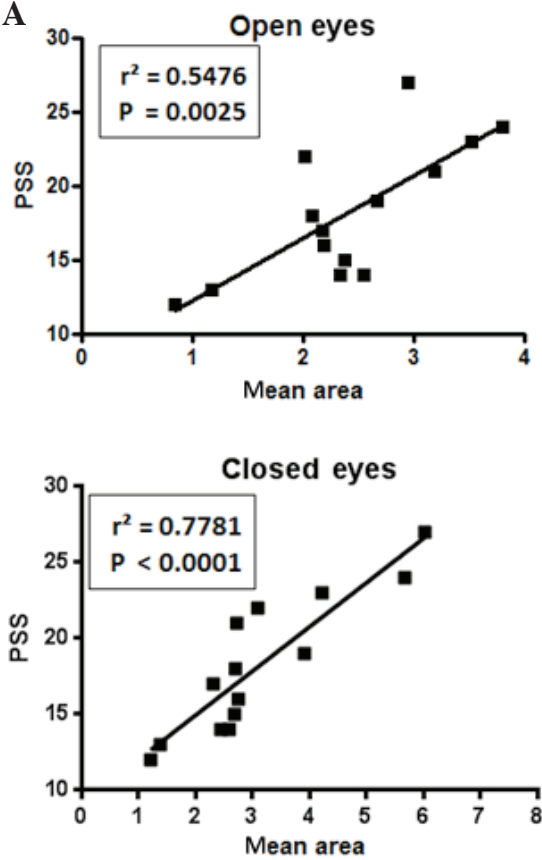

B

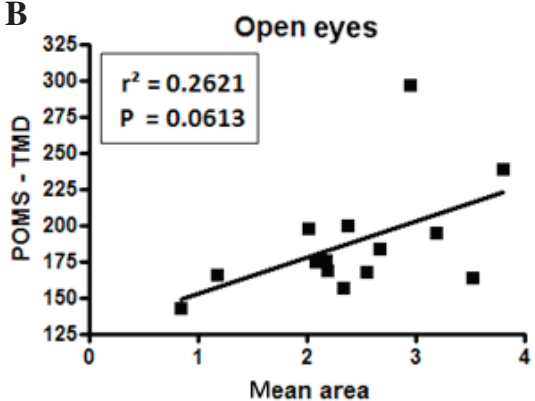

Closed eyes

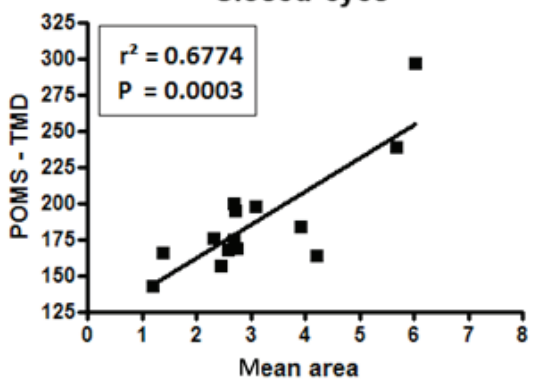

C

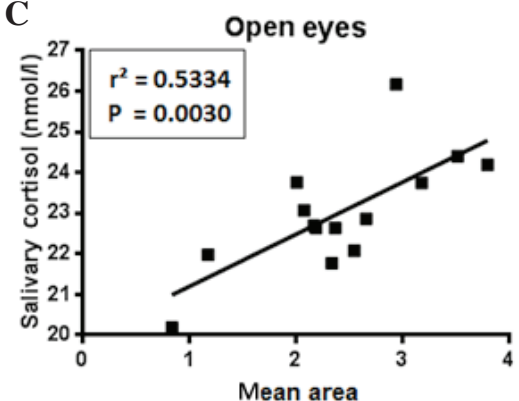

Closed eyes

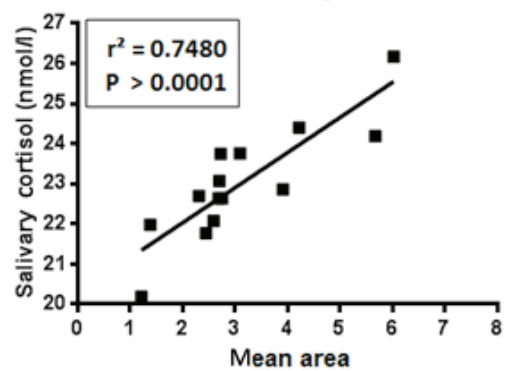

Figure 4. Correlations of the (A) perceived stress scale (PSS), (B) profile of mood states expressed as total mood disturbance (POMS - TMD) and (C) salivary cortisol with the mean $95 \%$ confidence ellipse area of COP (A95) value measured in balance position E with open and closed eyes for each subject. The mean A95 was significantly correlated with the PSS and salivary cortisol levels when eyes were both open and closed, but was only significantly correlated with POMS when eyes were open, not with closed eyes.

single subjects of the sample, as well as the mean values of the cortisol awakening profiles and the results of Tukey's multiple comparisons test between the mean values. In all studied subjects, there was a significant increase in the levels of salivary cortisol immediately after awakening (time 0 ), with levels reaching their maximum value after $30 \mathrm{~min}$. However, 
60 min after awakening, salivary cortisol concentration was not significantly different from that at time 0 .

There is a strong positive correlation between TMD, PSS and salivary cortisol. Fig. 3 illustrates the correlation between the POMS, expressed as TMD, the PSS and the salivary cortisol levels for each of the studied subjects. A strong significant positive correlation between these three measures was identified.

A95 varies greatly between participants. Regarding the postural control, Table II summarizes the results obtained when measuring (in $\mathrm{cm}^{2}$ ) the A95 in positions A-E (Fig. 1). A significant variation was observed between individuals, with the differences between 39 and $66 \%$ in the eyes open experiments and between 43 and $76 \%$ with eyes closed. Furthermore, when comparing the data from a single position measured with open eyes with those calculated with closed eyes, significant differences $(\mathrm{P}>0.05)$ were observed only in positions $\mathrm{D}$ and $\mathrm{E}$, with the worst values recorded in with eyes closed (Table II).

When comparing, for each subject, the scores of PSS, TMD and salivary cortisol with the A95 mean value measured in the position E (Fig. 4), a significant correlation was observed between the A95 and the salivary cortisol levels and the PSS with open and closed eyes. For POMS no significant correlation was observed when eyes were open, whereas a strong positive correlation was detected when eyes were closed.

\section{Discussion}

Previous evaluations of kinematic and kinetic variables have revealed their cognitive influences on gait and postural stability (32-36) and it has been observed that high levels of stress are associated with poorer executive function, abstract reasoning, processing speed, and visual-spatial memory (12-14). Furthermore, mood state is capable of influencing balance control (15) and anticipatory postural adjustments (16).

The main aim of the present study was to investigate the impact of stress on postural stability with or without visual input, whilst the secondary objective was to evaluate the effect of laterality.

The level of stress was measured with PSS whilst overall mood disturbance was assessed with POMS as TMD. Stress was also evaluated by measuring the rapid increase of free cortisol levels after awakening. Chronic stress exhibits an important role in the CAR but studies examining how chronic stress modulates the CAR have reported conflicting results (reviewed in 20,21). Certain studies observed a reduced CAR in association with chronic stress (37-41), whereas other studies described an increased CAR in individuals chronically exposed to stressful events (42-45).

Several elements have been suggested to explain these contrasting results. Fries et al (21) postulated that the duration of chronic stress may exert positive or negative influences on the CAR. This theory is supported by the results of a meta-analysis conducted by Miller et al (20), which concluded that 'timing is an especially critical element, as hormonal activity is elevated at stressor onset but reduces as time passes'. In the present study, a strong positive correlation was observed between CAR and psychological rating scales evaluating stress (PSS) or, more in general, mood states (POMS). Therefore, by accepting the conclusions of Miller et al (20), it is reasonable to assume that the subjects in the present study were exposed to relatively recent stressful conditions.

Postural stability was inferred from the A95 measured in five postural conditions, which were maintained for at least $52 \mathrm{sec}$ with open and closed eyes. A95 was selected as previous studies showed that this measure is the primary measure for postural stability $(28,29)$. Data regarding the COP indicated an increased significant body sway in two specific conditions, i.e. when eyes were closed in position $\mathrm{D}$ and $\mathrm{E}$, where the dominant foot was $10 \mathrm{~cm}$ behind the other. Therefore, when the two feet are not at the same level, maintenance of posture is easier when the dominant foot is ahead, regardless of visual input. This effect of laterality is in agreement with the observations of previous studies $(46,47)$.

In the present study, a strong correlation for each subject was observed between the PSS and TMD scores, as well as the levels of salivary cortisol and the mean value of A95 measured in the 5 positions when eyes were closed, whereas between the mean of A95 and PSS a significant correlation was only observed only with open eyes. It is worth noting that in previous studies aimed at evaluating the association between mood and postural control $(15,16)$ only the POMS was used. Although PSS and POMS co-vary linearly, on the basis of the results of the present study the former may be more appropriate than the latter to demonstrate heightened stress.

In conclusion, the present study demonstrated not only that the level of stress is capable of influencing postural stability, but also that this influence is particularly evident when no visual information is available during postural control.

\section{References}

1. Deschamps T, Magnard J and Cornu C: Postural control as a function of time-of-day: influence of a prior strenuous running exercise or demanding sustained-attention task. J Neuroeng Rehabil 10: 26, 2013.

2. Gribble PA, Tucker WS and White PA: Time-of-day influences on static and dynamic postural control. J Athl Train 42: 35-41, 2007.

3. Jorgensen MG, Rathleff MS, Laessoe U, Caserotti P, Nielsen OBF and Aagaard P: Time-of-day influences postural balance in older adults. Gait Posture 35: 653-667, 2012.

4. Paillard T: Effects of general and local fatigue on postural control: a review. Neurosci Biobehav Rev 36: 162-176, 2012.

5. Era P and Heikkinen E: Postural sway during standing and unexpected disturbance of balance in random samples of men of different ages. J Gerontol 40: 287-295, 1985.

6. Fernie GR, Gryfe CI, Holliday PJ and Llewellyn A: The relationship of postural sway in standing to the incidence of falls in geriatric subjects. Age Ageing 11: 11-16, 1982.

7. Laudani L, Casabona A, Perciavalle V and Macaluso A: Control of head stability during gait initiation in young and older women. J Electromyogr Kinesiol 16: 603-610, 2006.

8. Marsh AP and Geel SE: The effect of age on the attentional demands of postural control. Gait Posture 12, 105-113, 2000

9. Joormann J: Cognitive inhibition and emotion regulation in depression. Curr Dir Psychol Sci 19: 161-166, 2010.

10. McEwen BS and Stellar E: Stress and the individual. Mechanisms leading to disease. Arch Intern Med 153: 2093-2101, 1993.

11. Demirovic D and Rattan SI: Establishing cellular stress response profiles as biomarkers of homeodynamics, health and hormesis. Exp Gerontol 48: 94-98, 2013.

12. Arnsten AF: The biology of being frazzled. Science 280: 1711-1712, 1998 . 
13. Liston C, McEwen BS and Casey BJ: Psychosocial stress reversibly disrupts prefrontal processing and attentional control. Proc Natl Acad Sci USA 106: 912-917, 2009.

14. Oaten $\mathrm{M}$ and Cheng $\mathrm{K}$ : Academic examination stress impairs self-control. J Soc Clin Psychol 24: 254-279, 2005.

15. Bolmont B, Gangloff P, Vouriot A and Perrin PP: Mood states and anxiety influence abilities to maintain balance control in healthy human subjects. Neurosci Lett 329: 96-100, 2002.

16. Kitaoka K, Ito R, Araki H, Sei H and Morita Y: Effect of mood state on anticipatory postural adjustments. Neurosci Lett 370 65-68, 2004

17. Kirby RL, Price NA and MacLeod DA. The influence of foot position on standing balance. J Biomech 20: 423-427, 1987.

18. Cohen S, Kamarck T and Mermelstein R: A global measure of perceived stress. J Health Soc Behav 24: 385-396, 1983.

19. Pruessner J, Wolf O, Hellhammer D, Buske-Kirschbaum A, von Auer K, Jobst S, Kaspers F and Kirschbaum C: Free cortisol levels after awakening: a reliable biological marker for the assessment of adrenocortical activity. Life Sci 61: 2539-2549, 1997.

20. Miller GE, Chen E and Zhou ES: If it goes up, must it come down? Chronic stress and the hypothalamic-pituitary-adrenocortical axis in humans. Psychol Bull 133: 25-45, 2007.

21. Fries E, Dettenborn L and Kirschbaum C: The cortisol awakening response (CAR): facts and future directions. Int J Psychophysiol 72: 67-73, 2009.

22. Kudielka BM and Kirschbaum C: Sex differences in HPA axis responses to stress: a review. Biol Psychol 69: 113-132, 2005.

23. Oldfield RC: The assessment and analysis of handedness: the Edinburgh inventory. Neuropsychologia 9: 97-113, 1971.

24. Demitrack MA and Crofford LJ: Evidence for and pathophysiological implications of hypothalamic pituitary adrenal axis dysregulation in fibromyalgia and chronic fatigue syndrome. Ann NY Acad Sci 840: 684-697, 1998.

25. McNair DM, Lorr M and Dropplema LF: Manual for the profile of mood states. Education and Industrial Testing Service, San Diego, CA, 1971.

26. Terry PC and Lane AM: Normative values for the profile of mood states for use with athletic samples. J Appl Sport Psychol 12: 93-109, 2000.

27. Di Corrado D, Agostini T, Bonifazi M and Perciavalle V: Changes in mood states and salivary cortisol levels following two months of training in elite female water polo players. Mol Med Rep 9: 2441-2446, 2014

28. Boyle J, Danjou P, Alexander R, Calder N, Gargano C, Agrawa N, Fu I, McCrea JB and Murphy MG: Tolerability, pharmacokinetics and night-time effects on postural sway and critical flicker fusion of gaboxadol and zolpidem in elderly subjects. Br J Clin Pharm 67: 180-190, 2009

29. Norris V, Baisley KJ, Calder N, van Troostenburg AR and Warrington SJ: Assessment of the AccuSway ${ }^{\text {PLUS }}$ system in measuring the effect of lorazepam on body sway in healthy volunteers. Int J Pharm Med 19: 233-238, 2005.

30. Kudielka BM and Kirschbaum C: Awakening cortisol responses are influenced by health status and awakening time but not by menstrual cycle phase. Psychoneuroendocrinology 28: 35-47, 2003.
31. Curran-Everett D and Benos DJ: Guidelines for reporting statistics in journals published by the American Physiological Society. J Appl Physiol 97: 457-459, 2004.

32. Ebersbach G, Dimitrijevic MR and Poewe W: Influence of concurrent tasks on gait: A dual-task approach. Percept Mot Skills 81: 107-113, 1995.

33. Kang HG and Lipsitz LA: Stiffness control of balance during quiet standing and dual task in older adults: the MOBILIZE Boston Study. J Neurophysiol 104: 3510-3517, 2010.

34. Lajoie Y, Teasdale N, Bard C and Fleury M: Attentional demands for static and dynamic equilibrium. Exp Brain Res 97: 139-144, 1993.

35. Riley MA, Baker AA and Schmit JM: Inverse relation between postural variability and difficulty of a concurrent short-term memory task. Brain Res Bull 62: 191-195, 2003.

36. Vuillerme $\mathrm{N}$ and Vincent $\mathrm{H}$ : How performing a mental arithmetic task modify the regulation of centre of foot pressure displacements during bipedal quiet standing. Exp Brain Res 169: 130-134, 2006

37. Barker ET, Greenberg JS, Seltzer MM and Almeida DM: Daily stress and cortisol patterns in parents of adult children with a serious mental illness. Health Psychol 31: 130-134, 2012.

38. Buchanan TW, Kern S, Allen JS, Tranel D and Kirschbaum C: Circadian regulation of cortisol after hippocampal damage in humans. Biol Psychiatry 56: 651-656, 2004.

39. Meinlschmidt G and Heim C: Decreased cortisol awakening response after early loss experience. Psychoneuroendocrinology 30: 568-576, 2005.

40. Quevedo K, Johnson A, Loman M, Lafavor T and Gunnar M: The confluence of adverse early experience and puberty on the cortisol awakening response. Int J Behav Dev 36: 19-28, 2012.

41. Duan H, Yuan Y, Zhang L, Qin S, Zhang K, Buchanan TW and $\mathrm{Wu} \mathrm{J}$ : Chronic stress exposure decreases the cortisol awakening response in healthy young men. Stress 16: 630-637, 2013.

42. Gustafsson PE, Janlert U, Virtanen P and Hammarström A: The association between long-term accumulation of temporary employment, the cortisol awakening response and circadian cortisol levels. Psychoneuroendocrinology 37: 789-800, 2012.

43. Schlotz W, Hellhammer J, Schulz P and Stone AA: Perceived work overload and chronic worrying predict weekend-weekday differences in the cortisol awakening response. Psychosom Med 66: 207-214, 2004.

44. Steptoe A, Brydon L and Kunz-Ebrecht S: Changes in financial strain over three years, ambulatory blood pressure, and cortisol responses to awakening. Psychosom Med 67: 281-287, 2005.

45. Wust S, Federenko I, Hellhammer DH and Kirschbaum C: Genetic factors, perceived chronic stress, and the free cortisol response to awakening. Psychoneuroendocrinology 25: 707-720, 2000

46. Conson M, Mazzarella E and Trojano L: Self-touch affects motor imagery: a study on posture interference effect. Exp Brain Res 215: 115-122, 2011.

47. Mezaour M, Yiou E and Le Bozec S: Does symmetrical upper limb task involve symmetrical postural adjustments? Gait Posture 30: 239-244, 2009. 